\title{
Predictive monitoring for respiratory decompensation leading to urgent unplanned intubation in the neonatal intensive care unit
}

\author{
Matthew T. Clark1, Brooke D. Vergales ${ }^{2}$, Alix O. Paget-Brown², Terri J. Smoot ${ }^{3}$, Douglas E. Lake ${ }^{3,4}$, John L. Hudson', John B. Delos ${ }^{5}$, \\ John Kattwinkel ${ }^{2}$ and J. Randall Moorman ${ }^{3}$
}

BACKGROUND: Infants admitted to the neonatal intensive care unit (NICU), and especially those born with very low birth weight (VLBW; $<1,500 \mathrm{~g})$, are at risk for respiratory decompensation requiring endotracheal intubation and mechanical ventilation. Intubation and mechanical ventilation are associated with increased morbidity, particularly in urgent unplanned cases.

METHODS: We tested the hypothesis that the systemic response associated with respiratory decompensation can be detected from physiological monitoring and that statistical models of bedside monitoring data can identify infants at increased risk of urgent unplanned intubation. We studied 287 VLBW infants consecutively admitted to our NICU and found 96 events in 51 patients, excluding intubations occurring within $12 \mathrm{~h}$ of a previous extubation.

RESULTS: In order of importance in a multivariable statistical model, we found that the characteristics of reduced $\mathrm{O}_{2}$ saturation, especially as heart rate was falling; increased heart rate correlation with respiratory rate; and the amount of apnea were all significant independent predictors. The predictive model, validated internally by bootstrap, had a receiver-operating characteristic area of $0.84 \pm 0.04$.

CONCLUSION: We propose that predictive monitoring in the NICU for urgent unplanned intubation may improve outcomes by allowing clinicians to intervene noninvasively before intubation is required.

nfants born prematurely have extended stays in the neonatal intensive care unit (NICU). This is particularly true of infants born with very low birth weight (VLBW, $<1,500 \mathrm{~g}$ ), at least $65 \%$ of whom will require endotracheal intubation for the administration of mechanical ventilation (data for 2009-2010, from the Vermont Oxford Network of more than 900 centers, http://www.vtoxford.org/). These long ICU stays can be punctuated by clinical deterioration, including frequent apneas $(1,2)$ or other forms of respiratory decompensation, leading to urgent unplanned intubation, in which the infant is provided mechanical ventilation through an endotracheal tube.
In addition to worsening neonatal apnea, urgent unplanned intubation can result from sepsis, respiratory distress syndrome, pneumonia, exacerbation of chronic lung disease, or critical illness from conditions such as necrotizing enterocolitis. Although intubation for the purpose of mechanical ventilation is an effective intervention for respiratory decompensation, it is also associated with substantial morbidity and mortality, including pneumonia (3), barotrauma, and volutrauma leading to pneumothorax or bronchopulmonary dysplasia, and oxygen toxicity leading to pulmonary and retinal injury. Early detection of respiratory decompensation may allow for early and less obtrusive treatment, such as initiation or increase of noninvasive respiratory support such as continuous positive airway pressure, administration of bronchodilators, or evaluation and treatment of infection.

We hypothesize that some episodes of apparently sudden clinical deterioration in the NICU have precursors of altered control of heart rate and other physiological processes that require finely adaptive coupling among organs (4). This is the case with late-onset neonatal sepsis, in which reduced heart rate variability and transient decelerations can precede clinical signs of illness by $24 \mathrm{~h}(5-11)$. In a recent very large randomized clinical trial, we found that display of a multivariable statistical model that relates these abnormal heart rate characteristics (HRCs) to the fold increase in risk of sepsis in the next $24 \mathrm{~h}$ led to a more than $20 \%$ reduction in VLBW NICU mortality (12).

We have tested the hypothesis that respiratory decompensation leading to urgent unplanned intubations can also be preceded by changes apparent from bedside physiological monitoring. Similar to the development of the HRC index, or HeRO score, we have developed logistic regression models on the basis of physiological waveforms conventionally recorded in the NICU, including cardiac, respiratory, and pulse oximetry vital signs. Unlike the HeRO score, however, the new predictive model includes information from the respiratory as well as the cardiac system, and the interactions between the two. 


\section{RESULTS}

\section{Patient Population}

Times when no vital signs were recorded because of technical problems were excluded, leaving a population of 309 VLBW infants who had monitoring data available. Of the 309 VLBW infants with available data, data from 22 patients could be collected only when they were mechanically ventilated, and these patients were therefore excluded from the study. A total of 287 patients were included in the study. The demographic data of the patients in the study, and those of the subset of

Table 1. Demographic characteristics of the study population

\begin{tabular}{lc}
\hline All infants in the study $(N=287)$ & $27(25,29)$ \\
\hline EGA (wk) & 147 \\
Males & $1,010(783,1,268)$ \\
Birth weight (g) & $61(35,95)$ \\
Length of stay (d) & $15(2,37)$ \\
Ventilator days & $37(36,39)$ \\
PMA at discharge (wk) & \\
\hline Infants with events $(N=51)$ & 96 \\
\hline Events & 11 \\
Events due to sepsis & 32 \\
Males & $26(25,28)$ \\
EGA (wk) & $810(708,1,060)$ \\
Birth weight (g) & $97(67,107)$ \\
Length of stay (d) & $27(8,43)$ \\
Ventilator days & $39(37,41)$ \\
PMA at discharge (wk) & $29(26,31)$ \\
PMA at urgent unplanned intubation & \\
\hline Data are presented as median (25th, 75 th percentile). & \\
EGA, estimated gestational age; PMA, postmenstrual age. &
\end{tabular}

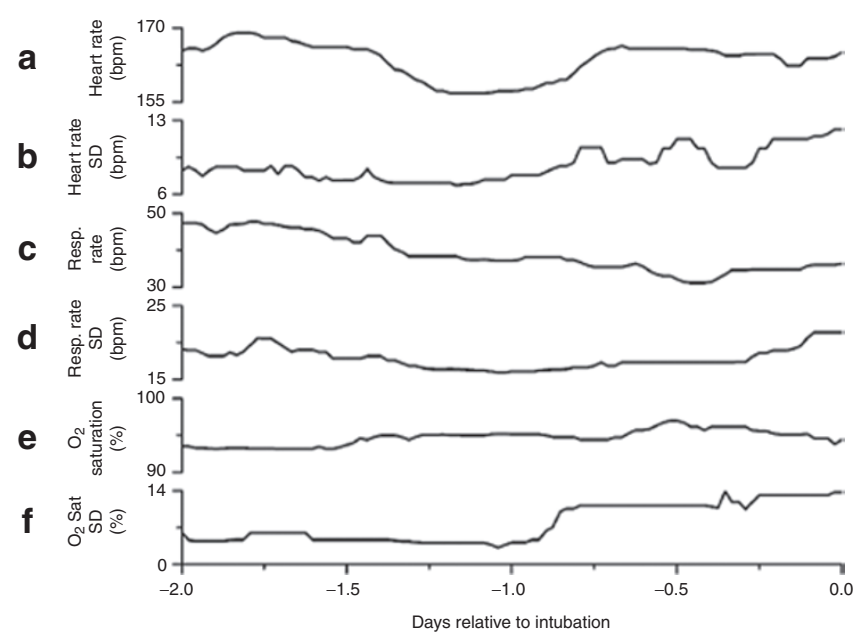

patients who had an urgent unplanned intubation, are shown in Table 1. In the population of 287 VLBW infants for whom data were recorded for at least $12 \mathrm{~h}$ before intubation, we found 96 unplanned intubation events in 51 patients.

\section{An Example of the Analysis}

Figure 1 shows the time series of cardiorespiratory parameters for an infant born with a birth weight of $1,460 \mathrm{~g}$ at $29 \mathrm{wk}$ estimated gestational age. This infant had an urgent unplanned intubation for respiratory acidosis $9 \mathrm{~d}$ after birth, and parameters are shown relative to the time of this event. The clinical goal is to identify patterns that are predictive of urgent unplanned intubations in VLBW infants.

The left column in Figure 1 shows the means and SDs of conventionally monitored vital signs, including heart rate, respiratory rate, and pulse oximetry level. During the time leading to intubation, this infant's physiological measurements present conflicting information. For example, the heart rate variability and arterial oxygen saturation are rising, consistent with improving status. Concurrently, the respiratory rate is falling and pulse oximetry variability is rising, consistent with deteriorating status.

The right column of Figure 1 shows correlations between the vital signs, as well as three measures of physiological stability: the level of cardiorespiratory coupling (13), duration of time spent in apnea with associated bradycardia and desaturation (or apnea burden) (14), and the output of a model for predicting neonatal sepsis on the basis of HRCs, the HeRO score (12). Correlations between heart and respiratory rates, and between heart rate and pulse oximetry, rise in the $2 \mathrm{~d}$ before urgent unplanned intubation. At the same time, the correlation between respiratory rate and pulse oximetry falls. The level of cardiorespiratory coupling $1 \mathrm{~d}$ before intubation is $25 \%$ of the value taken $2 \mathrm{~d}$ before intubation and falls nearly to zero by the

Figure 1. Time series of physiological measures for one patient before unplanned intubation. Unplanned intubation occurs at zero, on the right edge of the plots. During this time period, (a) mean heart rate dips, (b) heart rate SD increases, (c) mean respiratory rate decreases, (d) respiratory rate SD remains unchanged, (e) oxygen saturation remains unchanged, and (f) oxygen saturation SD increases. Moreover, during this time, (g) correlation between heart rate and respiratory rate increases, (h) correlation between heart rate and oxygen saturation increases, (i) correlation between respiratory rate and oxygen saturation decreases, (j) cardiorespiratory coupling decreases, (k) apnea burden increases, and (I) the HeRO score increases. bpm, beats per minute; cross-corr, cross-correlation; $\mathrm{HR}$, heart rate; $\mathrm{O}_{2}$ sat, oxygen saturation; Resp. rate, respiratory rate; $\mathrm{RR}$, respiratory rate. 
time of intubation. The patient's apnea burden is high throughout, and the HeRO score increases by fourfold over the day leading up to intubation.

Thus, the clinician has multiple streams of physiological data, all varying with time, interrelated to various degrees, and
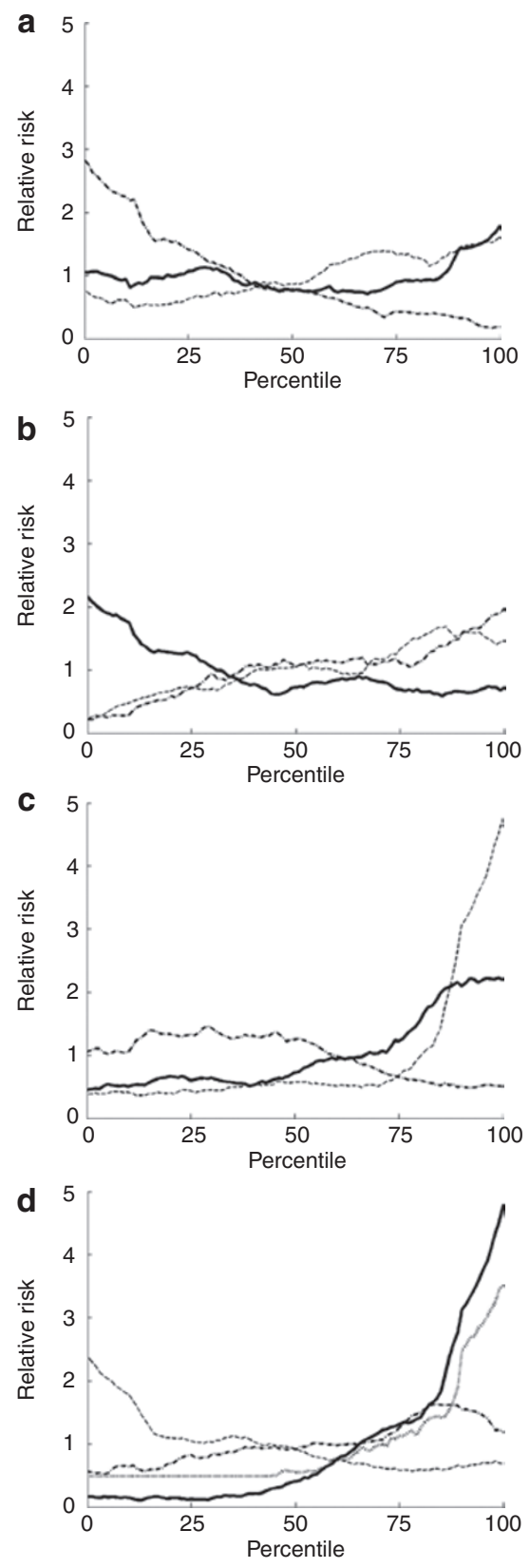

Figure 2. Relative risk of unplanned intubation in the next $24 \mathrm{~h}$ as a function of variable percentile. Percentiles are based on all values observed for a given variable, and variables are calculated over half-hour windows. (a) Relative risk vs. percentile of mean heart rate (solid line), respiratory rate (dashed line), and pulse oximetry (dashed-dotted line). (b) Relative risk vs. percentile of SD of heart rate (solid line), respiratory rate (dashed line), and pulse oximetry (dashed-dotted line). (c) Relative risk vs. percentile of correlation between heart rate and respiratory rate (solid line), heart rate and pulse oximetry (dashed line), and respiratory rate and pulse oximetry (dashed-dotted line). (d) Relative risk vs. percentile of the HeRO score (solid line), coupling (dashed line), fraction of beats during inhalation (dashed-dotted line), and apnea burden (gray dotted line). often with inconsistent trajectories. This justifies an approach using multivariate time series methods.

\section{Univariate Analyses}

Examination of patient records indicated that patient physiology undergoes changes before clinically relevant incidents (15) and urgent unplanned intubation, in particular. We exploit this fact by developing logistic regression models for intubation on the basis of physiological parameters. Figure 2 shows the relative risk of unplanned intubation in the next $24 \mathrm{~h}$ on the ordinate and the percentile of each physiological parameter on the abscissa. For example, the lowest and highest heart rates observed in our sample of infants are represented by the 0 and 100 percentiles, respectively. The nomenclatures $\mu_{i}$ and $\sigma_{i}$ indicate the mean and SD of vital sign $i$, respectively, and $\langle i j\rangle$ indicates the cross-correlation coefficient between vital signs $i$ and $j$ at zero lag. High respiratory and heart rates, and high respiratory and oxygenation variability are associated with increased risk of intubation, as is low oxygen saturation. Risk of intubation has a nonlinear relation with heart rate variability.

The curves in Figure 2 indicate the importance of each physiological parameter in predicting intubation. Parameters that provide a large dynamic range between risks at the 0 and 100 percentiles are good candidates for a model. Mean and variability of the pulse oximetry level, correlation between heart rate and pulse oximetry, cardiorespiratory coupling, and the HeRO score all have high dynamic ranges. The association of

Table 2. Performance of univariate logistic regression models for unplanned intubation

\begin{tabular}{|c|c|c|c|c|}
\hline Variable & $\mathrm{ROC}$ & $P$ value & Sign & $\chi^{2}$ \\
\hline \multicolumn{5}{|l|}{ Vital signs } \\
\hline$\mu_{\mathrm{HR}}$ & 0.53 & 0.18 & + & 0.2 \\
\hline$\sigma_{H R}{ }^{a}$ & 0.61 & 0.007 & + & 7.2 \\
\hline$\mu_{\mathrm{RR}}$ & 0.60 & 0.05 & + & 7.8 \\
\hline$\sigma_{\mathrm{RR}}$ & 0.61 & * & + & 13.2 \\
\hline$\mu_{\mathrm{spO2}}$ & 0.70 & * & - & 37.1 \\
\hline$\sigma_{\mathrm{SpO2}}$ & 0.62 & * & + & 13.0 \\
\hline \multicolumn{5}{|l|}{ Correlations } \\
\hline$<\mathrm{HRRR}>$ & 0.65 & * & + & 15.6 \\
\hline$<\mathrm{HRSpO}_{2}>$ & 0.74 & * & + & 47.5 \\
\hline$<\mathrm{RRSpO}_{2}>$ & 0.57 & 0.06 & - & 3.7 \\
\hline \multicolumn{5}{|c|}{ Physiological stability } \\
\hline Coupling & 0.62 & 0.03 & - & 4.8 \\
\hline CVC & 0.50 & 0.95 & - & $<0.1$ \\
\hline Beat fraction & 0.58 & 0.02 & + & 5.0 \\
\hline Apnea burden & 0.70 & * & + & 31.5 \\
\hline HeRO score & 0.81 & $*$ & + & 49.5 \\
\hline
\end{tabular}

CVC, cardioventilatory coupling; HR, heart rate; $R O C$, receiver-operating characteristic; $\mathrm{RR}$, respiratory rate; $\mathrm{SpO}_{2^{\prime}}$ peripheral oxygen saturation; $\mu$, mean; $\sigma, \mathrm{SD}$.

${ }^{a}$ A nonlinear transform was applied to heart rate variability before use in a logistic regression model. Specifically, the absolute difference between the measured heart rate variability and the median of all heart rate variability values were used. ${ }^{*} P<0.001$. 
HeRO is, in part, due to the 11 of 96 urgent unplanned intubation events in response to sepsis.

We deployed candidate predictor variables in a univariate logistic regression model to predict urgent unplanned intubation in the next $24 \mathrm{~h}$. Table 2 shows the performance of these models, including the receiver-operating characteristic, significance $(P$ value) and sign of the coefficient, and the goodness of fit $\left(\chi^{2}\right)$. To account for the nonlinear relation for heart rate variability, we recast it as the absolute difference between the variability and the median variability for all patients at all times. Univariate models were based only on the times when the parameter was available.

Heart rate itself had little predictive information. Low and, counterintuitively, high heart rate variability were both associated with upcoming unplanned intubation (16). The HeRO score had the best association with upcoming intubation, with receiver-operating characteristic area of 0.81 and $\chi^{2}$ value of 49 , followed by the cross-correlation of heart rate and $\mathrm{O}_{2}$ saturation ( 0.74 and 48 , respectively). The former reflects the reduced heart rate variability and transient decelerations that can accompany early phases of neonatal sepsis, and the latter reflects the coordinated bradycardia and $\mathrm{O}_{2}$ desaturation that accompany neonatal apneas.

\section{Multivariable Analysis}

We used the parameters in which univariate coefficients were significant (Table 2, $P \leq 0.05$ ) as inputs to a multivariate logistic

Table 3. Performance of multivariate model for unplanned intubation

\begin{tabular}{lccccc}
\hline Variable & $\begin{array}{c}\text { Coefficient } \\
\text { (normalized) }^{\mathrm{a}}\end{array}$ & Coefficient & SE & Pvalue & $\chi^{2}$ \\
\hline$\mu_{\mathrm{SpO2}}$ & -0.03 & -0.13 & 0.02 & $*$ & 32.1 \\
$<\mathrm{HRRR}>$ & 24.98 & 3.51 & 0.70 & 0.017 & 24.9 \\
$<\mathrm{HRSPO}_{2}>$ & 23.24 & 3.48 & 0.64 & $*$ & 30.1 \\
$\begin{array}{l}\text { Apnea } \\
\text { burden }\end{array}$ & 0.009 & 0.04 & 0.02 & 0.02 & 5.1 \\
\hline
\end{tabular}

$\mathrm{HR}$, heart rate; $\mathrm{RR}$, respiratory rate; $\mathrm{SpO}_{2^{\prime}}$ peripheral oxygen saturation; $\mu$, mean. aCoefficients normalized by SD of the variable. ${ }^{*} P<0.001$. regression model, 11 in total for 96 events. We determined this to be acceptable, as meaningful multivariate models are known to require 6-10 events per predictor (17). During periods when a parameter was not available, the median value of that parameter (for all patients at all times) was used. Parameters in which multivariate regression coefficients did not reach significance were eliminated from the model, and a new model was created. We note that the HeRO score, the best-performing individual predictor, did not make the final model. The HeRO score does not add information to models that include the correlation between heart rate and pulse oximetry: the two measures have a moderate correlation $(r=0.45)$. Table 3 shows the coefficients and SEs for each parameter used in the final model. The area under the receiver-operating curve for the final model is $0.84 \pm 0.04$ as determined by bootstrapping $(5,18)$.

The output of the multivariate logistic regression model is the probability of urgent unplanned intubation in the next 24 h. Figure 3 (left) shows the model output for 96 events in 51 patients, normalized to the relative risk by dividing by the average rate of intubation in the next $24 \mathrm{~h}(0.5 \%)$. The median increases by two-thirds during the day before intubation, and the median output $12 \mathrm{~h}$ before intubation is significantly higher than that $36 \mathrm{~h}$ before intubation (gray dots, $P=0.001$ ).

Figure 3 (right) shows the probability (solid) that the null hypothesis of the Wilcoxon signed-rank test is true, i.e., that the median model output $t$ days before intubation is equal to the corresponding median model output $t-1.5 \mathrm{~d}$ before intubation. The cutoff for rejecting the null hypothesis $(P=0.05)$ is shown as the dashed horizontal line. Model outputs throughout the 24-h period before urgent unplanned intubation are significantly higher than model outputs 36-60 h before intubation.

\section{Internal Validation}

Bootstrapping showed the $95 \%$ confidence interval to be \pm 0.04 (18).

Implementation of the Predictive Model for an Individual Patient Figure 4 shows the relative risk for the patient whose records are shown in Figure 1 on the basis of our multivariate logistic
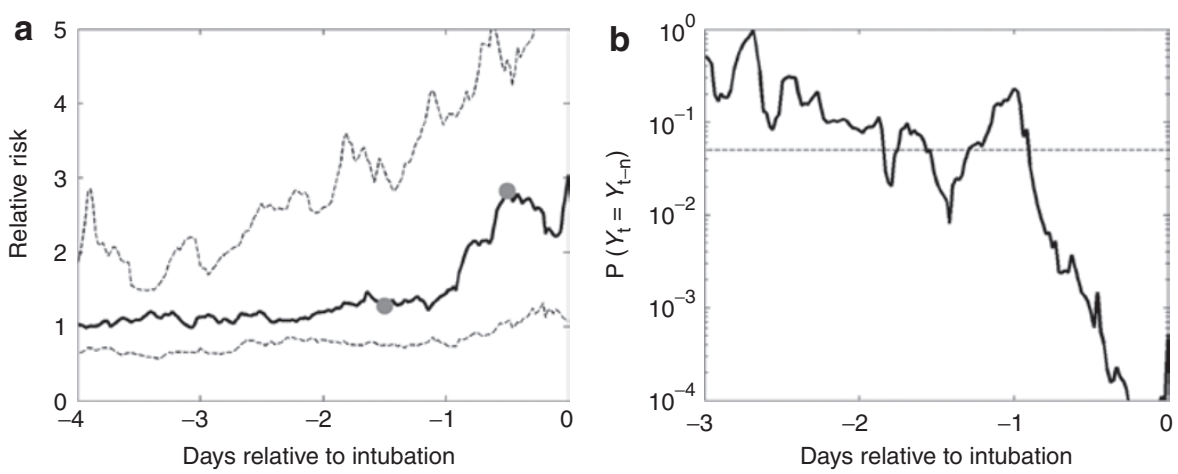

Figure 3. Model output for the patient sample relative to time of intubation. (a) Median (solid) and $25 \%$, $75 \%$ (dashed) model output for 96 urgent unplanned intubation events in 51 patients. The median model output $12 \mathrm{~h}$ before intubation is significantly higher than the output $36 \mathrm{~h}$ before intubation (gray dots, $P=0.001$ based on a signed-rank test). (b) The probability that the null hypothesis of the Wilcoxon signed-rank test is true. Paired data are separated by $36 \mathrm{~h}(n=36)$. The dashed line shows the cutoff for rejecting the null hypothesis $(P=0.05)$. Model outputs in the day before intubation are significantly higher than the values $36 \mathrm{~h}$ before intubation. 


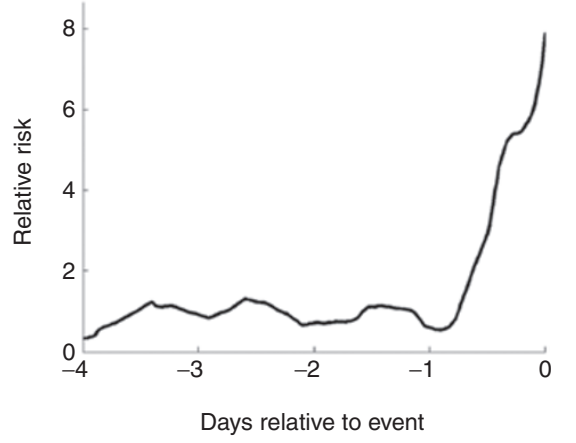

Figure 4. Relative risk of urgent unplanned intubation for the patient shown in Figure 1 based on the multivariate logistic regression model defined in Table 3. From $1 \mathrm{~d}$ before unplanned intubation to the time of intubation, the estimated risk increases eightfold.

regression model (Table 3). The relative risk increases fivefold from 24 to $12 \mathrm{~h}$ before the event.

\section{DISCUSSION}

We studied changes in bedside physiological monitoring parameters in premature infants at risk for respiratory decompensation. We used conventional and cross-correlation measures based on vital signs, novel variables based on cardiorespiratory waveforms, and multivariate logistic regression to predict episodes of urgent unplanned intubation. Our predictive statistical model has good performance, with a receiver-operating characteristic area of 0.84 , and allowed the identification of characteristics that added independent information to one another after taking them all into account. The clinical characteristics of the decompensating infant include, in order of decreasing predictive importance on the basis of goodness of fit, low $\mathrm{O}_{2}$ saturation, coincident fluctuations in heart rate and $\mathrm{O}_{2}$ saturation, correlated heart rate and respiratory rate, and increasing apneas. The heart rate-based HeRO score, which had the highest univariate predictive performance with receiver-operating characteristic area of 0.81 , was displaced in the final model by these other parameters, all of which incorporate information about respiration.

The value of these findings is in the possibility of bedside predictive monitoring for neonatal respiratory decompensation. The strengths of this analysis are that we used data that are conventionally available in the NICU and require no new sensors or contact with the infant. A limitation is that the model is not yet externally validated. In addition, further studies should investigate the impact that changing respiratory support and medication administration may have on the model output.

Inputs to the multivariate model listed in Table 3 were selected to optimize model performance and fit to the data. The significance of physiological parameters, including those not in the multivariate model, provides insight into mechanisms underlying clinical decompensation leading to the need for urgent unplanned intubation. The importance of mean and variability of pulse oximetry, and correlation between pulse oximetry and heart rate as well as heart rate and respiratory rate, indicates a role for hypoxemia. The association of increased HRC index with intubation indicates a decline in cardiac control through extracellular signaling (19) and the autonomic nervous system $(20,21)$. Decreased cardiorespiratory coupling is an indicator of critical deterioration, in agreement with the concept of systemic inflammatory response syndrome in adult patients (15).

The modern age of high-speed data analysis allows great opportunities for synthesizing the large number of data streams available to the intensive care clinician. Although the insights into the clinical picture of the decompensating infant from this study are not surprising, there is potentially great value in bedside predictive monitoring that is constantly available, requires no new contact with the infant, and optimally leverages data that are already present. Such monitoring could never replace the clinical judgment of experienced doctors and nurses; however, when considering an apparently stable infant in a busy NICU, a rising risk score might place the clinician at the right bedside at the right time.

\section{METHODS}

\section{Patient Population}

We collected cardiorespiratory waveforms and vital signs from 1,438 consecutive admissions to the University of Virginia NICU from January 2009 through June 2011. For the 320 VLBW infants, we also collected demographic data including admission and discharge dates, types and times of respiratory support, nursing documentation of apnea and bradycardia, and disposition and status at discharge. Times when no vital signs were recorded because of technical problems were excluded. The University of Virginia Institutional Review Board gave permission for this study with waiver of consent status.

\section{Definition of Urgent Unplanned Intubation}

Urgent unplanned intubations were defined as nonelective initiation of mechanical ventilation. Accepted causes included worsening respiratory status from primary lung disease, increasing apnea, respiratory acidosis, and increasing requirement for inspired oxygen. There is no protocol in the University of Virginia NICU that defines when to intubate for these causes. Decisions are made on a case-by-case basis when less-invasive treatment (e.g., continuous positive airway pressure) proves ineffective. Often, intubations occur overnight on the basis of need as perceived by the NICU staff, and by their nature are considered urgent and unplanned.

We excluded planned intubations before surgery or other elective procedures, protocol-driven surfactant administration requiring $<12 \mathrm{~h}$ of intubation, and 19 instances of reintubation within $12 \mathrm{~h}$ of a prior extubation. These clinically important events were excluded because they do not provide $12 \mathrm{~h}$ of nonventilated data on which to develop a model. Two clinical experts independently investigated patient records for each intubation, and only events deemed by both reviewers to meet our criteria for urgent unplanned intubation were included.

\section{Data Collection}

Vital signs and waveforms were collected from all bedside monitors in our 45-bed NICU by a centralized server (BedmasterEx, Excel Medical, Jupiter, FL) behind the clinical firewall. Vital signs (heart rate, respiratory rate, and pulse oximetry) were calculated by the monitor by averaging over the previous $10 \mathrm{~s}$ and were collected every $2 \mathrm{~s}$. Waveforms included signals from three electrocardiogram leads digitized at $240 \mathrm{~Hz}$, chest impedance pneumograph digitized at 60 $\mathrm{Hz}$, and oximetry plethysmography digitized at $120 \mathrm{~Hz}$. Data were transferred to our parallel computing and storage cluster. All infants had continuous HeRO monitoring (Medical Predictive Science, Charlottesville, VA) and HRC indexes (12) were collected hourly.

\section{Data Analysis}

Calculations were made on 30-min blocks of data collected during periods of spontaneous ventilation, i.e., when the infant was not 
receiving mechanical ventilation. As candidate predictors, we calculated the mean and SD of each vital sign-heart rate, respiratory rate, and $\mathrm{O}_{2}$ saturation-along with the cross-correlation of each vital sign with the others. From the continuous waveforms-electrocardiogram, chest impedance, and oximetry plethysmography - we calculated more complex physiological and statistical measures: cardiorespiratory coupling, cardioventilatory coupling, fraction of heartbeats during inhalation, apnea burden, and the HeRO score.

Cardiorespiratory coupling (hereafter referred to as "coupling") and fraction of heartbeats during inhalation were calculated every 30 s over the previous $4 \mathrm{~min}$ when data were of sufficient quality for analysis (13). Coupling is preferential alignment of heartbeats within the respiratory cycle, and this phenomenon was defined as epochs for which the distribution of heartbeats within the respiratory cycle had $<0.1 \%$ chance of occurring from random numbers (13). Each half hour, the fraction of measures that exhibited coupling was calculated and averaged over the previous $12 \mathrm{~h}$. The fraction of beats during inhalation was defined as the mean over the previous $12 \mathrm{~h}$. Cardioventilatory coupling is the preferential alignment of inhalation to the heartbeat and was calculated every $30 \mathrm{~s}$ over the previous $10 \mathrm{~min}$. We defined cardioventilatory coupling as epochs for which the relationship between each inhalation and the previous R-wave had less than a $5 \%$ chance of occurring from noise given the number of intervals $(22,23)$. Each half hour, the fraction of measures that exhibited cardioventilatory coupling was calculated and averaged over the previous $12 \mathrm{~h}$.

Cardiorespiratory waveforms were automatically analyzed to detect central apnea using the methods of Lee et al. (14). Briefly, breathing cessations were detected as low-variance epochs in the chest impedance pneumograph after notch filtering in heart-clock time to eliminate cardiac artifact and high-pass filtering to remove movement artifact. Heartbeats were detected using a threshold-based algorithm (24) as implemented by Clifford and co-workers $(25,26)$. Apneas were defined as breathing cessation of at least $10 \mathrm{~s}$ with associated bradycardia (heart rate $<100$ beats per minute) and desaturation (peripheral oxygen saturation $<80 \%)(14,27)$. Apnea burden was defined as the number of seconds that the infant was apneic during the previous $12 \mathrm{~h}$.

HRC indexes were collected from monitors in the NICU each hour, and values were carried over to the subsequent half hour. The HRC index is an output of a logistic regression model based on the RR interval SD, sample asymmetry, and sample entropy that detects reduced variability and transient decelerations and reports the fold increase in the risk of neonatal sepsis in the next $24 \mathrm{~h}$. It has been externally validated and has been shown to add information to the laboratory tests and clinical signs, and to reduce mortality when displayed (4-12).

\section{Development and Internal Validation of Logistic Regression Models}

We used these conventional and novel physiological variables as inputs to logistic regression models. Measurements within the $24 \mathrm{~h}$ before an urgent unplanned intubation event were labeled as an outcome of 1 and used as events to be predicted. All other measurements (excluding data before failed extubation) were labeled as outcome 0 . Standard maximum likelihood estimation was used to determine the coefficients for the logistic regression model (28). This approach corrects both for unequal variances and correlated responses from individual patients. More specifically, estimates of regression coefficients and other parameters of the model are obtained in a standard fashion, but the $P$ values are corrected using the "sandwich" estimator of SEs (29). For internal validation, we used a cluster bootstrap technique whereby 1,000 new samples of the same size were obtained by resampling the infants with replacement (30). The 2.5 and 97.5 percentiles of the sample of risks are used as lower and upper limits for a $95 \%$ confidence interval, respectively.

The multivariate predictive model for urgent unplanned intubation was developed by first creating univariate models for each conventional and novel physiological variable on the basis of all available data for that variable. Variables that yielded statistically significant models (as defined by coefficients with $P<0.05$ ) were incorporated into a multivariate model. Variables whose coefficients were not significant in the multivariate model were removed. Not all parameters could be measured at all times, and we replaced missing data with the median value for all measurements of that variable from all patients when creating the multivariate model. Values were imputed in this way to allow the model to be calculated as often as possible, with the trade-off of decreasing the accuracy of the final model.

\section{STATEMENT OF FINANCIAL SUPPORT}

This work was funded by National Institutes of Health grant no. 1RC2HD064488.

Disclosure: D.E.L. and J.R.M. wish to disclose financial interest in Medical Predictive Science Corporation (Charlottesville, VA).

\section{REFERENCES}

1. Darnall RA, Ariagno RL, Kinney HC. The late preterm infant and the control of breathing, sleep, and brainstem development: a review. Clin Perinatol 2006;33:883-914; abstract $\mathrm{x}$.

2. Barrington K, Finer N. The natural history of the appearance of apnea of prematurity. Pediatr Res 1991;29:372-5.

3. Apisarnthanarak A, Holzmann-Pazgal G, Hamvas A, Olsen MA, Fraser VJ. Ventilator-associated pneumonia in extremely preterm neonates in a neonatal intensive care unit: characteristics, risk factors, and outcomes. Pediatrics 2003;112:1283-9.

4. Fairchild KD, O'Shea TM. Heart rate characteristics: physiomarkers for detection of late-onset neonatal sepsis. Clin Perinatol 2010;37:581-98.

5. Griffin MP, O'Shea TM, Bissonette EA, et al. Abnormal heart rate characteristics preceding neonatal sepsis and sepsis-like illness. Pediatr Res 2003;53:920-6.

6. Griffin MP, Lake DE, Bissonette EA, et al. Heart rate characteristics: novel physiomarkers to predict neonatal infection and death. Pediatrics 2005;116:1070-4.

7. Griffin MP, O'Shea TM, Bissonette EA, et al. Abnormal heart rate characteristics are associated with neonatal mortality. Pediatr Res 2004;55:782-8.

8. Griffin MP, Lake DE, Moorman JR. Heart rate characteristics and laboratory tests in neonatal sepsis. Pediatrics 2005;115:937-41.

9. Griffin MP, Lake DE, O'Shea TM, Moorman JR. Heart rate characteristics and clinical signs in neonatal sepsis. Pediatr Res 2007;61:222-7.

10. Kovatchev BP, Farhy LS, Cao H, et al. Sample asymmetry analysis of heart rate characteristics with application to neonatal sepsis and systemic inflammatory response syndrome. Pediatr Res 2003;54:892-8.

11. Moorman JR, Delos JB, Flower AA, et al. Cardiovascular oscillations at the bedside: early diagnosis of neonatal sepsis using heart rate characteristics monitoring. Physiol Meas 2011;32:1821-32.

12. Moorman JR, Carlo WA, Kattwinkel J, et al. Mortality reduction by heart rate characteristic monitoring in very low birth weight neonates: a randomized trial. J Pediatr 2011;159:900-6.e1.

13. Clark MT, Rusin CG, Hudson JL, et al. Breath-by-breath analysis of cardiorespiratory interaction for quantifying developmental maturity in premature infants. J Appl Physiol 2012;112:859-67.

14. Lee $H$, Rusin CG, Lake DE, et al. A new algorithm for detecting central apnea in neonates. Physiol Meas 2012;33:1-17.

15. Godin PJ, Buchman TG. Uncoupling of biological oscillators: a complementary hypothesis concerning the pathogenesis of multiple organ dysfunction syndrome. Crit Care Med 1996;24:1107-16.

16. Griffin MP, Scollan DF, Moorman JR. The dynamic range of neonatal heart rate variability. J Cardiovasc Electrophysiol 1994;5:112-24.

17. Griffin MP, Lake DE, Bissonette EA, et al. Heart rate characteristics: novel physiomarkers to predict neonatal infection and death. Pediatrics 2005;116:1070-4.

18. Feng Z, McLerran D, Grizzle J. A comparison of statistical methods for clustered data analysis with Gaussian error. Stat Med 1996;15:1793-1806.

19. Küster $\mathrm{H}$, Weiss $M$, Willeitner $\mathrm{AE}$, et al. Interleukin-1 receptor antagonist and interleukin- 6 for early diagnosis of neonatal sepsis 2 days before clinical manifestation. Lancet 1998;352:1271-7.

20. Ellenby MS, McNames J, Lai S, et al. Uncoupling and recoupling of autonomic regulation of the heart beat in pediatric septic shock. Shock 2001;16:274-7.

21. Buchman TG, Stein PK, Goldstein B. Heart rate variability in critical illness and critical care. Curr Opin Crit Care 2002;8:311-5.

22. Larsen PD, Galletly DC. Cardioventilatory coupling in heart rate variability: the value of standard analytical techniques. Br J Anaesth 2001;87:819-26. 
23. Tzeng YC, Larsen PD, Galletly DC. Mechanism of cardioventilatory coupling: insights from cardiac pacing, vagotomy, and sinoaortic denervation in the anesthetized rat. Am J Physiol Heart Circ Physiol 2007;292:H1967-77.

24. Pan J, Tompkins WJ. A real-time QRS detection algorithm. IEEE Trans Biomed Eng 1985;32:230-6.

25. Li Q, Mark RG, Clifford GD. Robust heart rate estimation from multiple asynchronous noisy sources using signal quality indices and a Kalman filter. Physiol Meas 2008;29:15-32.

26. Tarassenko L, Clifford G, Townsend N. Detection of ectopic beats in the electrocardiogram using an auto-associative neural network. Neural Proc Lett 2001;14:15-25.
27. Finer NN, Higgins R, Kattwinkel J, Martin RJ. Summary proceedings from the apnea-of-prematurity group. Pediatrics 2006;117: S47-51.

28. White H. Maximum-likelihood estimation of mis-specified models. Econometrica 1982;50:1-25.

29. Wei LJ, Lin DY, Weissfeld L. Regression-analysis of multivariate incomplete failure time data by modeling marginal distributions. J Am Stat Assoc 1989;84:1065-73.

30. Steyerberg EW, Harrell FE Jr, Borsboom GJ, et al. Internal validation of predictive models: efficiency of some procedures for logistic regression analysis. J Clin Epidemiol 2001;54:774-81. 